\title{
Análise da Influência do Parâmetro de Hurst na Avaliação de Desempenho de Filas P/M/1
}

\author{
Wiliam H. Hisatugu \& Anilton S. Garcia
}

\begin{abstract}
Resumo - O uso de sistemas de filas com Distribuição de Pareto é uma das formas de tratar o tráfego autosimilar em redes de pacotes. Existem vários trabalhos desenvolvidos sobre o uso da distribuição de Pareto para a modelagem de tráfego. Uma dessas propostas especifica uma relação explícita entre a Distribuição de Pareto e o parâmetro de Hurst através do parâmetro $\alpha$. Outros trabalhos estudam o impacto da manipulação dos parâmetros da Distribuição de Pareto na modelagem de filas. Essa manipulação ocasiona a existência de várias representações para a Distribuição de Pareto. Dentre as várias representações, a mais utilizada é conhecida como Distribuição de Pareto com Dois Parâmetros. Este trabalho propõe que seja utilizada a representação conhecida como Distribuição de Lomax para o tratamento de tráfego autosimilar. Através de pesquisa bibliográfica, resultados analíticos e numéricos, este trabalho demonstra que a Distribuição de Lomax é mais adequado que a Distribuição de Pareto com Dois Parâmetros na modelagem de tráfego, e que é importante que o valor do parâmetro $\beta$ seja também estimado usando o parâmetro de Hurst.
\end{abstract}

Palavras chaves - Distribuição de Lomax, Distribuição de Pareto, Sistemas de Filas, Tráfego autosimilar, Parâmetro de Hurst

Abstract - The Pareto Distribution is a way normally used to treat selfsimilar traffic modeling. There are many works about the using of Pareto Distribution in traffic modeling. Among these works, exists a propose of relationship between Pareto Distribution and Hurst parameter with $\alpha$ parameter. Through the parameter handling is possible to obtain different representations for the Pareto Distribution and the called Two Parameters Pareto is used so much. This work proposes the using of a representation called Lomax Distribution to model selfsimilar traffic. With bibliografica review, analytical and numerical results, this work demonstrates that Lomax Distribution is more realistic than Two Parameters Pareto Distribution in queueing systems modeling, and is very

Wiliam H Hisatugu é aluno de doutorado do Programa de Pós Graduação em Engenharia Elétrica da Universidade Federal do Espírito Santo; membro do Grupo de Pesquisa Wimax-UFES; Suas áreas de pesquisa são: Planejamento de Capacidade e Avaliação de Desempenho de Redes de Telecomunicações Planejamento de Capacidade de Redes Móveis de Banda Larga (Wimax Móvel), Pesquisa Operacional/Ciência da Gestão, Enterprise Architecture, Análise Quantitativa e Qualitativa; E-mail: hisatugu@ele.ufes.br

Anilton S Garcia é membro do Programa de Pós Graduação em Engenharia Elétrica da Universidade Federal do Espírito Santo; coordenador do Grupo de Pesquisa Wimax/UFES; Suas áreas de interesse são Gerência Integrada de Redes e Serviços através do desenvolvimento de ferramentas computacionais Agentes Inteligentes para Aplicações em Redes e Gerência de Redes, Ferramentas de Avaliação de Desempenho e Planejamento de Capacidade em Redes Multimídia, Redes Ópticas e de Redes BWA (Broadband Wireless Access) com foco em aspectos de QoS e Mobilidade em Redes WiMax. E-mail:anilton@inf.ufes.br

Programa de Pós Graduação em Engenharia Elétrica, Centro Tecnológico da Universidade Federal do Espírito Santo - Av. Fernando Ferrari, s/n - Campus Universitário Goiabeiras - 29060-900 Vitória/ES important to estimate $\beta$ parameter using Hurst parameter. Keywords - Lomax Distribution, Pareto Distribution, Queueing Systems, Selfsimilar Traffic, Hurst Parameter

\section{INTRODUÇ̃̃o}

A Distribuição de Pareto, por ser uma função de cauda pesada, é bastante utilizada na modelagem de tráfego em redes de pacotes, que tem características autosimilares [1]. Ela pode assumir várias representações de acordo com a manipulação dos seus três parâmetros [2]: decaimento $\alpha$, escala $\beta$ e deslocamento $\gamma$. Em todas as representações conhecidas é mantida a característica de cauda pesada [2]. Contudo, a intensidade de decaimento de sua função de densidade de probabilidade é alterada devido a manipulação dos seus parâmetros. Dentre as representações conhecidas, a representação chamada Distribuição de Pareto com Dois Parâmetros [3] tem sido bastante utilizada para a modelagem de tráfego de redes de pacotes. Nesta representação, o parâmetro de deslocamento $\gamma$ tem o mesmo valor que o parâmetro de escala $\beta$.

Entretanto, os resultados numéricos obtidos em [3] indicam, sem provas formais, que o parâmetro de deslocamento $\gamma$ influencia diretamente no desempenho do modelo de filas que utiliza a Distribuição de Pareto. Esses indicadores numéricos apontam que a Distribuição de Pareto com Dois Parâmetros pode não ser realista o bastante para o tratamento de tráfego em redes de pacotes. Em [2], é sugerido que a representação conhecida como Distribuição de Lomax é mais realista que a distribuição de Pareto com Dois Parâmetros.

A relação entre a Distribuição de Pareto e o parâmetro de Hurst, H, também é um tópico bastante relevante na modelagem de tráfego autosimilar. Em [4] é mostrada uma relação entre $\mathrm{H}$ e a Distribuição de Pareto estimando o parâmetro $\alpha$ em função de $\mathrm{H}$. Essa relação tem sido bastante utilizada nos trabalhos que modelam o tráfego em redes e consideram o grau de autosimilaridade do tráfego através do parâmetro de Hurst, como em [5] e [6].

Todavia, o parâmetro $\beta$ também influencia na intensidade do decaimento da função de densidade de probabilidade (pdf) da Distribuição de Pareto. Um estudo realizado em [7] propõe uma forma de estimar o parâmetro $\beta$ também em função de $\mathrm{H}$ para a Distribuição de Lomax.

Este trabalho tem por objetivos: mostrar que o parâmetro $\gamma$ influencia o desempenho do tráfego modelado e, assim, que a Distribuição de Lomax é mais adequada para a modelagem de tráfego que a Distribuição de Pareto com Dois Parâmetros; 
mostrar que a relação entre $\beta$ e o parâmetro de Hurst também deve ser explicitamente especificada para a melhor modelagem do tráfego autosimilar.

Para o desenvolvimento deste trabalho foi feita uma pesquisa bibliográfica, de onde foi constatada, inicialmente, a maior utilização da Distribuição de Pareto com Dois Parâmetros [3]. Essa pesquisa bibliográfica também mostrou que a escolha da representação da Distribuição de Pareto influencia na modelagem de sistemas de filas, as propostas de estimação dos parâmetros $\alpha$ e $\beta$ em função de H. Dessa pesquisa, foram analisadas as equações da média das Distribuições de Lomax e de Pareto com Dois Parâmetros. Essa análise mostrou que a diferença entre os valores das médias é sempre $\gamma$, mostrando que a Distribuição de Lomax é mais realista que a Distribuição de Pareto com Dois Parâmetros. Com as informações obtidas da pesquisa bibliográfica e da análise das equações das médias são feitas análises numéricas no modelo de filas $\mathrm{G} / \mathrm{M} / 1$ [8], onde a função de distribuição genérica $\mathrm{G}$ é a Distribuição de Pareto. Os resultados indicam como a diferença entre as médias das duas representações estudadas neste trabalho se comportam no modelo de filas G/M/1, onde a Distribuição de Pareto com Dois Parâmetros gera um tempo de espera inferior ao da Distribuição de Lomax. Os resultados também recomendam fortemente que seja estabelecido uma relação explícita entre o parâmetro $\beta$ e o parâmetro de $H$.

Para a melhor compreensão do trabalho desenvolvido, este artigo é organizado nas seguintes seções: na seção II é feita a revisão bibliográfica sobre a modelagem de tráfego com a Distribuição de Pareto; na seção III é feita a análise das equações das médias da Distribuição de Lomax e da Distribuição de Pareto com Dois Parâmetros; na seção IV são feitas análises comparativas sob duas perspectivas, a primeira sobre a relação explícita entre o parâmetro de Hurst e a Distribuição de Pareto, e a segunda sobre o resultado obtido na seção III; na seção V são apresentadas as conclusões do trabalho realizado neste artigo.

\section{Trabalhos Relacionados a Modelagem de Tráfego usando a Distribuição de Pareto}

A Distribuição de Pareto é bastante utilizada em várias áreas, como por exemplo: análise econômica, confiabilidade de hardware e teoria de filas [2]. Nas redes de telecomunicações, ela tem sido usada extensivamente na modelagem de tráfego de redes de pacotes, que possui características autosimilares [5][6][7]. A caracterização da Distribuição de Pareto é feita através da definição de seus três parâmetros: decaimento $\alpha$, escala $\beta$ e deslocamento $\gamma$. A manipulação desses três parâmetros possibilita o uso de diferentes representações da Distribuição de Pareto. A manipulação dos parâmetros e a escolha da representação da Distribuição de Pareto são temas de vários trabalhos. Especificamente em sistemas de filas, este trabalho destaca o trabalho descrito em [3] que faz uma comparação do desempenho da Distribuição de Pareto com Um Parâmetro com a Distribuição de Pareto com Dois Parâmetros. Outra representação usada na modelagem de tráfego é a Distribuição de Lomax. As funções de densidade de probabilidade da Distribuição de Pareto com Um Parâmetro, da Distribuição de Pareto com Dois Parâmetros e da Distribuição de Lomax são apresentadas respectivamente em (1), (2) e (3).

$$
\begin{gathered}
f(x)=\frac{\alpha}{(x+1)^{\alpha+1}} \\
(x \geqslant 0),(\alpha>0),(\beta=1),(\gamma=0) \\
f(x)=\frac{\alpha \gamma^{\alpha}}{(x)^{\alpha+1}} \\
(x \geqslant \gamma),(\alpha>0),(\beta=\gamma),(\gamma>0) \\
f(x)=\frac{\alpha \beta^{\alpha}}{(\beta+x)^{\alpha+1}} \\
(x \geqslant 0),(\alpha>0),(\beta>0),(\gamma=0)
\end{gathered}
$$

Quando aplicadas a modelagem de tráfego, cada uma dessas representações geram diferentes resultados e têm diferentes comportamentos. Os resultados numéricos obtidos em [3] mostram que a Distribuição de Pareto com Dois Parâmetros tem um tempo de espera na fila menor que o da Distribuição de Pareto com Um Parâmetro, quando os modelos de filas são submetidos a uma mesma carga $\rho$. Sem o uso de demonstrações formais, [3] sugere que esse menor tempo de espera deve-se ao parâmetro de deslocamento $\gamma$, indicando que a Distribuição de Pareto com Dois Parâmetros não é realista o suficiente para o tratamento de filas.

Outra questão bastante importante sobre a aplicação da Distribuição de Pareto em modelagem de tráfego é a sua relação com o parâmetro $\mathrm{H}$ que é usado para definir o grau de autosimilaridade de um fenômeno. Uma relação entre a Distribuição de Pareto e H é desenvolvida em [4], onde é proposta uma forma de estimar o parâmetro de decaimento $\alpha$ em função de H. Esta relação é apresentada em (4).

$$
\alpha=3-2 \mathrm{H} \quad 0.5<H<1
$$

Esta relação é bastante usada em trabalhos que usam a Distribuição de Pareto para modelar o tráfego autosimilar através da definição do valor de $\mathrm{H}$. A restrição mostrada para os valores possíveis para $\mathrm{H}$ faz com que o parâmetro $\alpha$ tenha como limite superior o valor 2 e limite inferior o valor 1 . Isto significa que, quando a relação descrita em (4) é usada para tratar o tráfego autosimilar com a Distribuição de Pareto, a média existe e a variância é infinita.

Contudo, o decaimento não é definido somente por $\alpha$, conforme pode ser observado na Fig 1. O parâmetro $\beta$ também influencia na intensidade do decaimento, ou seja, quanto menor seu valor, mais delgada é a curva da pdf, e mantendo a característica de cauda pesada. 


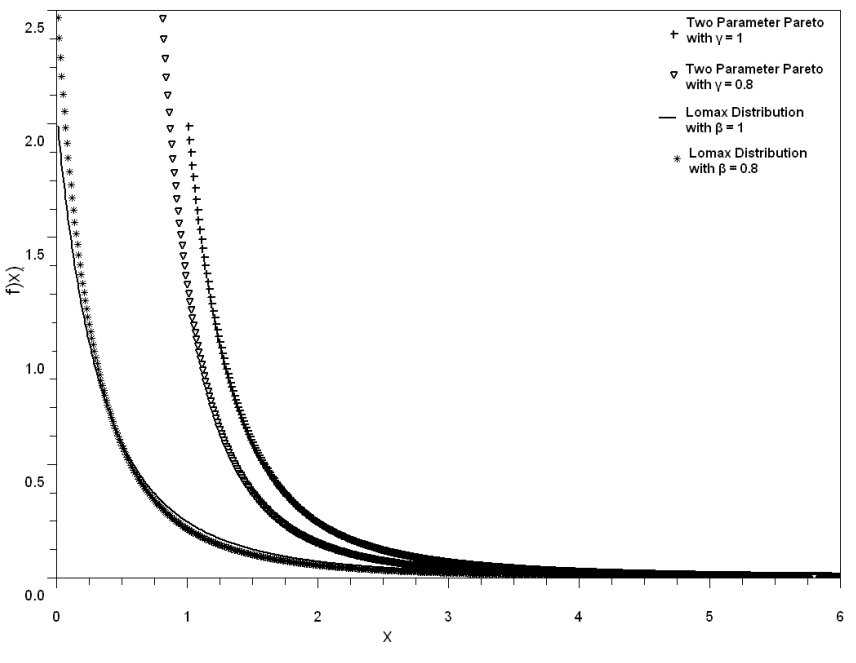

Fig. 1 - Exemplos de Gráficos da pdf da Distribuição de Pareto com $\alpha=$ 2.05. Observa-se que o Parâmetro $\beta$ influencia no decaimento da pdf da Distribuição de Pareto

Como a intensidade do decaimento também é influenciado pelo parâmetro $\beta$, este trabalho assume que é preciso considerar uma relação explícita entre $\beta$ e $\mathrm{H}$. O trabalho desenvolvido em [7] define uma equação para estimar o parâmetro $\beta$ em função do parâmetro de Hurst, que é ilustrada em (5).

$$
\begin{gathered}
\beta=-3.3181 \mathrm{H}^{2}+4.4567 \mathrm{H}-1.141 \\
0.5<H<1
\end{gathered}
$$

As restrições ao parâmetro $\alpha$ mostradas em [4] são mantidas em [7]. A equação (5) foi desenvolvida a partir de uma técnica de interpolação de pontos para vários valores de $\beta$ na Distribuição de Lomax. As análises realizadas em [7] recomendam que o parâmetro $\beta$ também deve ser estimado em função de $\mathrm{H}$. Contudo, a revisão bibliográfica deste trabalho indica que a Distribuição de Lomax é menos usada que as outras duas representações. De acordo com [2], a Distribuição de Lomax é mais adequada que a Distribuição de Pareto com Dois Parâmetros na modelagem de filas.

Para fins de comparação, a Tabela 1 mostra os valores obtidos da média para a Distribuição de Pareto com Dois Parâmetros com $\gamma=1$ [6] e com $\alpha$ definido em função de $H$, e para a Distribuição de Lomax com $\alpha$ e $\beta$ definidos em função de H, conforme é apresentado em [7]. Em [6], a Distribuição de Pareto com Dois Parâmetros é usada para simular o tráfego TCP (Transmission Control Protocol). Os resultados apresentados em [6] indicam a melhor adequação da Distribuição de Pareto com Dois Parâmetros na modelagem de fenômenos autosimilares do que a distribuição exponencial. Contudo, a análise realizada em [6] não faz nenhuma análise em modelos de filas. Em [6], $\beta$ é definido em função de $\gamma$, e este último é escolhido como o valor mínimo inicial submetido a função de densidade de probabilidade de Pareto.
TABELA I

Diferença dos Valores para a Média para a Distribuição de Lomax e a Distribuiç̃a de Pareto com Dois Parâmetros

\begin{tabular}{|c|c|c|}
\hline H & $\begin{array}{c}\text { Média obtida para } \\
\text { Distribuição de Lomax }\end{array}$ & $\begin{array}{c}\text { Média obtida para a Distribuição de } \\
\text { Pareto com Dois Parâmetros }\end{array}$ \\
\hline 0.55 & 0.3405108 & 2.1111111 \\
\hline 0.60 & 0.42313 & 2.25 \\
\hline 0.65 & 0.5056539 & 2.4285714 \\
\hline 0.70 & 0.588035 & 2.6666667 \\
\hline 0.75 & 0.6701875 & 3 \\
\hline 0.80 & 0.75194 & 3.5 \\
\hline 0.85 & 0.8328925 & 4.3333333 \\
\hline 0.90 & 0.911845 & 6 \\
\hline 0.95 & 0.9827975 & 11 \\
\hline
\end{tabular}

A Tabela 1 mostra ainda que existe uma grande diferença quando se usa a abordagem proposta em [6] e quando se usa a abordagem proposta em [7]. Pelos valores obtidos, é esperado que, à medida que o tráfego tem um grau de autosimilaridade maior, maior é o valor da média.

Contudo, existe a média aumenta de forma muito mais acentuado no modelo proposto em [6] com a Distribuição de Pareto com Dois Parâmetros, do que no modelo proposto em [7]. Os resultados apresentados na Tabela 1 fortalecem o argumento de que deve ser investigada a relação entre o parâmetro $\beta$ e H, e verificar a adequação da proposta feita em [7] na modelagem de filas.

Face aos resultados observados na Tabela 1 e na revisão bibliográfica, na próxima seção é feita uma análise nas equações da média da Distribuição de Lomax e da Distribuição de Pareto com Dois Parâmetros com ênfase no parâmetro $\gamma$.

III. Análise das Equações da Média da Distribuição de Lomax e da Distribuição de Pareto com Dois Parâmetros

O principal objetivo desta seção é mostrar que o tempo de espera em filla quando é utilizada a Distribuição de Pareto com Dois Parâmetros é menor devido ao parâmetro $\gamma$. Assim, esta seção também busca provas formais quanto a indicação apresentada em [2] de que a Distribuição de Lomax é mais realista que a Distribuição de Pareto com Dois Parâmetros na modelagem de filas. Para fazer esta demonstração, é feita uma análise das equações das médias de ambas as distribuições. Para existir o primeiro momento é preciso que o parâmetro $\alpha$ seja maior que 1 . A equação para estimativa do parâmetro $\alpha$ em função de $\mathrm{H}$ (4) satisfaz esta restrição. As equações das médias da Distribuição de Lomax $\left(M_{\text {lomax }}\right)$ e da Distribuição de Pareto com Dois Parâmetros ( $M_{\text {Pareto com Dois }}$ Parâmetros) são ilustradas respectivamente em (6) [7] e (7) [3].

$$
M_{\text {lomax }}=\frac{\beta}{\alpha-1}
$$




$$
M_{\text {Paretocom Dois Parâmetros }}=\frac{\alpha \gamma}{\alpha-1}
$$

Analisando as expressões (6) e (7), quando o valor de $\beta$ em (6) for o mesmo que em $\gamma$ em (7), o resultado de (7) é o mesmo obtido em (6) multiplicado por $\alpha$.

No intuito de fazer uma análise formal mais detalhada optou-se por verificar a diferença entre (7) e (6) adotando-se $\beta=\gamma$. O desenvolvimento dessa diferença é descrito em (8),

$$
\begin{gathered}
M_{\text {Paretocom Dois Parâmetros }}-M_{\text {lomax }} \\
\frac{\alpha \gamma}{\alpha-1}-\frac{\beta}{\alpha-1}, \quad \beta=\gamma \\
\frac{\alpha \gamma}{\alpha-1}-\frac{\gamma}{\alpha-1}=\frac{\gamma(\alpha-1)}{\alpha-1}=\gamma \\
M_{\text {Paretocom Dois Parâmetros }}-M_{\text {lomax }}=\gamma
\end{gathered}
$$

O resultado final de (8) indica que diferença entre as médias é $\gamma$. Este resultado mostra que a premissa apresentada em [3] quanto a influência de $\gamma$ no tempo de espera está correta. Ele também reforça a premissa descrita em [2] de que a Distribuição de Lomax é mais realista de a Distribuição de Pareto com Dois Parâmetros em modelos de filas.

A equação (8) também é um indicio de que o parâmetro $\beta$ deve ser estimado em função de $\mathrm{H}$, quando se deseja usar a Distribuição de Pareto para modelar o tráfego autosimilar, pois $\beta$, juntamente $\operatorname{com} \alpha$, influenciam na intensidade do decaimento da pdf de Pareto e o parâmetro $\gamma$

Dada as restrições impostas por (4), não é relevante verificar outras características relacionadas aos momentos de outras ordens das duas representações da Distribuição de Pareto. Entretanto, o resultado final de (8) juntamente com os resultados obtidos pelo trabalho desenvolvido em [3] e em [7] é um forte indício de que o parâmetro de deslocamento tem causa um melhor desempenho de modelos de filas que usam a Distribuição de Pareto com Dois Parâmetros.

A próxima seção explora os efeitos da diferença mostrada em (8) em modelos de fila $\mathrm{G} / \mathrm{M} / 1$, quando $\mathrm{G}$ é uma representação da Distribuição de Pareto.

IV. Avaliação de Desempenho de Modelo de Fila P/M/1 usando a Distribuição de Lomax e a Distribuição de Pareto com Dois PARÂMETROS

Nesta seção são feitas considerações acerca dos resultados observados neste trabalho, em [3] e em [7] em modelos de filas $\mathrm{P} / \mathrm{M} / 1$, onde P é a Distribuição de Pareto. As análises são feitas através das equações relacionadas ao tempo de espera na fila de modelo $\mathrm{G} / \mathrm{M} / 1$, descritos em [8].

Esta análise é feita através do tempo médio de espera na fila. A equação para obtenção do tempo médio de espera na fila é descrita em [8], e ilustrada em (9).

$$
W_{q}=\frac{\sigma}{\mu(1-\sigma)}
$$

onde $\sigma$ é a única raiz de (10), que possui valor menor que 1, e $\mathrm{A}^{*}$ é a Transformada de Laplace do tempo médio entre as chegadas.

$$
\sigma=A^{*}[\mu(1-\sigma)]
$$

Quando o tempo médio entre chegadas é definido por uma Distribuição de Pareto, não existe uma forma fechada para a sua Transformada de Laplace. Contudo, existem formas de obter uma aproximação da Transformada de Laplace para a Distribuição de Pareto. Para este trabalho é utilizada a técnica chamada TAM (Transform Approximation Method) modificada, que é proposta em [9]. A análise do modelo de fila é divida em duas partes: na primeira, é feita uma análise comparativa entre o modelo usado em [6] com a Distribuição de Pareto com Dois Parâmetros, estimando $\alpha$ em função de $\mathrm{H}$ e fixando $\gamma$ em 1, e o modelo proposto em [7], usando a Distribuição de Lomax com $\alpha$ e $\beta$ sendo estimados em função de $\mathrm{H}$; na segunda, a Distribuição de Pareto com Dois Parâmetros é comparada com Distribuição de Lomax usando vários valores para $\beta$ e $\gamma$. Essa segunda análise busca verificar os efeitos de (8) nos tempos de espera da fila em modelo $\mathrm{P} / \mathrm{M} / 1$.

\section{A. Análise 1}

Nesta subseção é feita uma comparação em modelo de fila $\mathrm{G} / \mathrm{M} / 1$ usado em [6] e o proposto em [7]. Em [6], é utilizada a Distribuição de Pareto com Dois Parâmetros, onde $\alpha$ é estimado em função de H, conforme (4), e $\gamma$ fixado em 1. Em [7], é usada a Distribuição de Lomax com $\alpha$ e $\beta$ estimados em função de $\mathrm{H}$ usando (4) e (5). Cabe ressaltar que devido ao valores dos parâmetros escolhidos, as formas das pdfs das duas representações são diferentes. O objetivo é analisar o desempenho das duas propostas quando são submetidos ao mesmo valor de $\mathrm{H}$ e carga $\rho$.

Aplicando (9) e (10) para obter os tempos médio de espera, Wq, para uma carga $\rho=0.8$, obtêm-se os resultados apresentados na Tabela II.

Os resultados observados na Tabela II revelam que para o exemplo adotado, a Distribuição de Pareto com Dois Parâmetros, onde somente $\alpha$ é obtido em função de $\mathrm{H}$, tem desempenho inferior ao da Distribuição de Lomax, onde tanto $\alpha$ como $\beta$ são obtidos em função de $\mathrm{H}$, conforme proposto em [7]. Em ambos os modelos, os tempos de espera aumentam juntamente com o parâmetro de Hurst. As diferenças entre os valores usados na Distribuição de Pareto com Dois Parâmetros com $\gamma$ igual a 1 e a Distribuição de Lomax com $\alpha$ e $\beta$ em função de $H$ são indicadores de que o parâmetro $\beta$ deve ser estimado em função de H. A Fig 2 ilustra os resultados da Tabela II. 
TABELA II

Tempo Médio de Espera em Fila para a Distribuição de Lomax e para a Distribuição de Pareto com Dois Parâmetros no Modelo P/M/1 $\rho=0.8$ E $\gamma=1$

\begin{tabular}{|c|c|c|c|}
\hline $\mathrm{H}$ & $\beta$ & $\begin{array}{c}\text { Wq para Pareto com Dois } \\
\text { Parâmetros }\end{array}$ & $\begin{array}{c}\text { Wq para a Distribuição } \\
\text { de Lomax }\end{array}$ \\
\hline 0.55 & 0.3064597 & 6.9239363 & 3.5621724 \\
\hline 0.60 & 0.338504 & 8.601618 & 5.1593683 \\
\hline 0.65 & 0.3539577 & 11.341636 & 7.5210866 \\
\hline 0.7 & 0.352821 & 16.337085 & 11.427093 \\
\hline 0.75 & 0.3350937 & 27.080408 & 19.059976 \\
\hline 0.80 & 0.300776 & 57.440964 & 38.409602 \\
\hline 0.85 & 0.2498677 & 200.93585 & 117.8949 \\
\hline 0.9 & 0.182369 & 2534.8825 & 1139.0768 \\
\hline 0.95 & 0.0982797 & Não obtido & 26207.147 \\
\hline
\end{tabular}

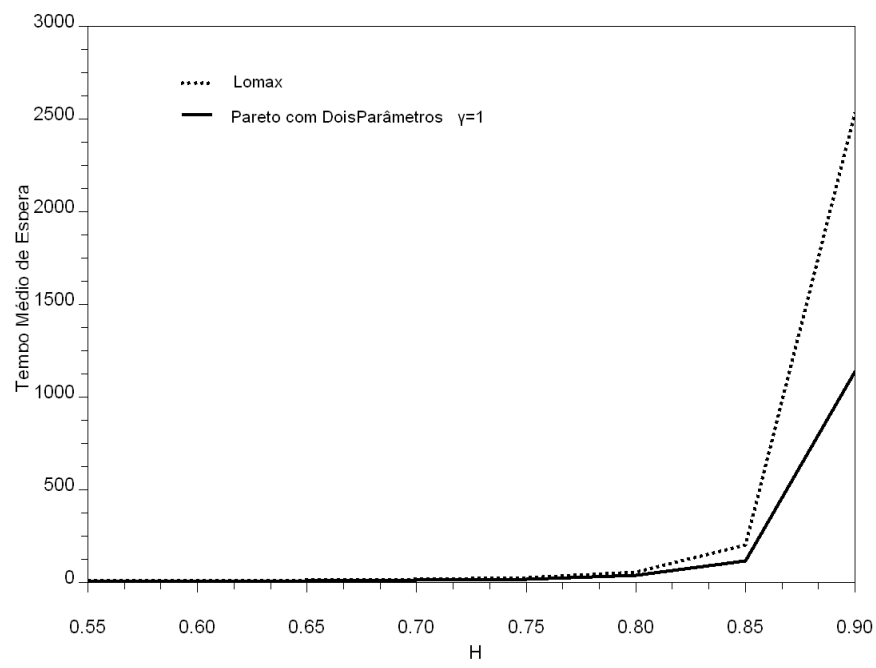

Fig 2 - Tempo de Espera x Parâmetro de Hurst: Distribuição de Lomax e Distribuição de Pareto com Dois Parâmetros

$\mathrm{Na}$ próxima subseção é feita uma análise sobre a influência do resultado observado em (9) em modelos de fila $\mathrm{P} / \mathrm{M} / 1$, onde $1<\alpha<2$.

\section{B. Análise 2}

Nesta segunda análise, é feita uma comparação entre os tempos de espera na filla entre a Distribuição de Pareto com Dois Parâmetros e a Distribuição de Lomax, quando $1<\alpha<2$. O objetivo desta análise é verificar o comportamento da fila $\mathrm{P} / \mathrm{M} / 1$ sob as condições que ocasionaram o resultado de (8), e verificar o impacto de (8) no comportamento de filas $\mathrm{P} / \mathrm{M} / 1$.

As condições para esta análise é que a carga $\rho=0.8, \beta=\gamma$ e $\mathrm{H}=0.7$, ocasionando um $\alpha=1.6$. Cabe ressaltar que, neste caso, as formas das pdfs para as duas representações são as mesmas deslocadas do parâmetro $\gamma$. Os resultados do tempo médio de espera na fila são apresentados na Tabela III. A Fig 3 apresenta os resultados da Tabela III.
TABELA III

Tempo Médio de Espera na Fila para a Distribuição de Lomax e a Distribuição de Pareto com Dois Parâmetros no Modelo P/M/1 $\rho=0.8$ and $\alpha=1.6$

\begin{tabular}{|c|c|c|}
\hline$\beta$ & $\begin{array}{c}\text { Wq para Pareto com Dois } \\
\text { Parâmetros }\end{array}$ & $\begin{array}{c}\text { Wq para a Distribuição de } \\
\text { Lomax }\end{array}$ \\
\hline 0.1 & 1.6335488 & 3.2387799 \\
\hline 0.2 & 3.2674168 & 6.4775579 \\
\hline 0.3 & 4.9011255 & 9.718897 \\
\hline 0.4 & 6.5341951 & 12.95853 \\
\hline 0.5 & 8.1685424 & 16.198161 \\
\hline 0.6 & 9.8012917 & 19.437794 \\
\hline 0.7 & 11.43484 & 22.671456 \\
\hline 0.8 & 13.069668 & 25.917058 \\
\hline 0.9 & 14.703377 & 29.156691 \\
\hline
\end{tabular}

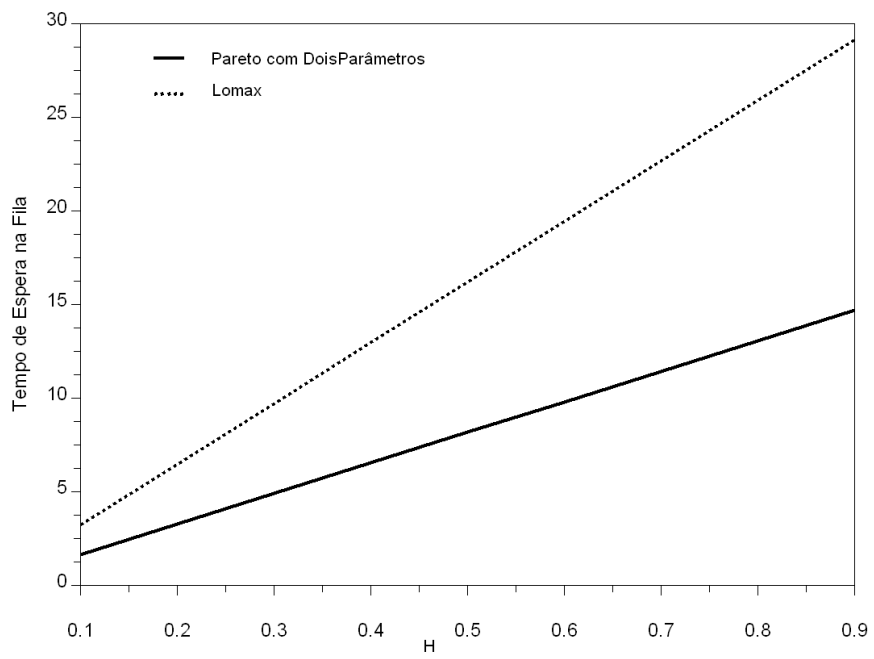

Fig 3 - Tempo Médio de Espera em Fila: Distribuição de Lomax X Distribuição de Pareto com Dois Parâmetros

Ao contrário do exemplo apresentado na Tabela II, os resultados a Tabela III indicam que a Distribuição de Pareto com Dois Parâmetros tem um menor tempo de espera na fila que a Distribuição de Lomax. Este resultado já era esperado, uma vez que o tempo médio entre chegadas na Distribuição de Lomax é menor do que na Distribuição de Pareto com Dois Parâmetros, gerando como conseqüência uma taxa de chegadas maior, função do parâmetro de deslocamento $\gamma$.

No entanto, em situações reais, onde se deve considerar a chegadas a partir do instante $t=0$, a equação (8), juntamente com a Tabela III, mostra que a Distribuição de Lomax é mais adequada que a Distribuição de Pareto com Dois Parâmetros. Esta premissa é baseada na definição da pdf da Distribuição de Pareto com Dois Parâmetros, onde o início do seu esboço é definido por $\gamma$, conforme é observado na Fig. 1.

$\mathrm{Na}$ seção $\mathrm{V}$ são descritas as conclusões feitas neste trabalho. 


\section{Conclusẽo}

Este trabalho investigou sobre os efeitos do parâmetro $\gamma$ e da relação entre a Distribuição de Pareto com o parâmetro de Hurst. O trabalho mostra de forma analítica como o parâmetro $\gamma$ tem influência sobre o desempenho de uma fila $\mathrm{P} / \mathrm{M} / 1$ através da análise numérica da equação da média, e que a Distribuição de Lomax é mais adequada que a Distribuição de Pareto com Dois Parâmetros. A pesquisa bibliográfica constatou que predominantemente, a relação entre a Distribuição de Pareto e o parâmetro de Hurst é definida unicamente pelo parâmetro $\alpha$. É feita uma comparação entre uma proposta usando a Distribuição de Pareto com Dois Parâmetros com $\gamma$ fixado em 1 e uma proposta de uso da Distribuição de Lomax, onde tanto $\alpha$ como $\beta$ são estimados em função de H. Nessa comparação é mostrado que a proposta que usa a Distribuição de Lomax tem tempos de espera em fila inferiores que a proposta que usa a Distribuição de Pareto com Dois Parâmetros.

Dessa maneira, este trabalho conclui que: a Distribuição de Lomax é mais adequada que a Distribuição de Pareto com Dois Parâmetros; esta melhor adequação da Distribuição de Lomax deve-se ao fato de que nesta representação, o parâmetro $\gamma$ tem valor igual a 0 ; como o parâmetro $\beta$ influencia no decaimento da pdf da Distribuição de Pareto, ele também deve ser estimado em função de $\mathrm{H}$.

Como proposta de trabalho futuro recomenda-se que: seja verificado a aderência da proposta de estimação do parâmetro $\beta$ em função de $\mathrm{H}$ através do monitoramento e modelagem de um tráfego real e da comparação com outras técnicas de estimativas de parâmetros da Distribuição de Pareto.

\section{Agradecimentos}

Os autores agradecem aos professores Dr. Magnus Martinello e Dr. Rodrigo Varejão pelas valiosas discussões acerca do trabalho desenvolvido.

\section{REFERÊNCIAS}

[1] Gordon J; "Pareto Process as a Model of Self-Similar Packet Traffic"; Proceedings of IEEE Global Telecommunications Conference 1995 , Vol. 3, pp. 2232 - 2236; 1995

[2] Hastings N A J, Peacock J B; "Statistical Distributions"; London: Butterworth and Co Ltda; 1975

[3] Shortle J F, Fischer M J, Gross D, Masi D M B; “One-Parameter Pareto, Two-Parameter Pareto, Three-Parameter Pareto: Is there a Modeling Difference?"; The Telecommunications Review 2005; pp 79-91; 2005

[4] Adie R G, Zukerman M, Neame T D; "Broadband Traffic Modeling. Simple Solutions for Hard Problems"; IEEE Communications Magazine, Vol 36, Issue 8, pp 88-95, August 1998;

[5] Xie M, Ye M, Feng S, Song H; "Performance of a Queuing Model With Pareto Input Traffic For Wireless Network Nodes"; Proceedings of International Conference in Wireless Communications, Networking and Mobile Computing 2005, Vol2, pp 867-870; 2005;

[6] Shu-Gang L, Pei-Jin W, Lin-Ji Q; "Modeling and Simulation of SekfSimilar Data Traffic"; Proceedings of the Fourth International Conference on Machine Learning and Cybernetics, Guangzhou, pp 3921-3925; August 2005

[7] Prata Junior O G, Garcia A S; "Uma proposta de modelagem matemática para tratamento de tráfego com característica autosimilar";XXII Simpósio Brasileiro de Redes de Computadores SBRC 2004, Gramado; 2004

[8] Kleinrock L; “Queueing Systems - Vol 1: Theory"; John Wiley \& Sons Inc.; 1975.

[9] Shortle J F, Fischer M J, Gross D, Masi D M B; "Using the Transform Approximation Method to Analyze Queues"; Journal of Probability and Statistical Science, vol 1, no. 1, pp 15-27; 2003; 